\title{
Macrobenthic Pollution Bioindicator for Ecological Monitoring in Riverine Ecosystem
}

\author{
Shreya Roy, Canciyal Johnson, Supriti Bayen, Trupti Rani Mohanty, Archisman Ray, Manisha Bhor, Himanshu \\ Sekhar Swain and Basanta Kumar Das*
}

ICAR-Central Inland Fisheries Research Institute, India

Submission: February 02, 2022; Published: February 18, 2022

*Corresponding author: Basanta Kumar Das, ICAR- Central Inland Fisheries Research Institute, Barrackpore, Kolkata 700120 , India

\begin{abstract}
Freshwater riverine systems are facing series of stress due to different anthropogenic activities, eutrophication and geomorphological alterations. Pollution is the current ecological hazard leading to the deterioration in river water quality and thereby modifying the quantitative distribution of the inhabiting organisms. In the present study, four major eco-physicochemical parameters are selected to correlate with the available benthic species distributed in the entire stretch of river Ganga, India. Total nitrogen, total phosphate, total chlorophyll and soil organic carbon are considered to be the primary indicators of water and soil pollution and hence, contemplated for correlation with benthic distribution. Out of the total recorded 69 species from river Ganga, 13 species belonging to three different phyla viz. Mollusca, Arthropoda and Annelida was observed to be influenced $(\mathrm{p} \leq 0.05)$ by the aforesaid pollution indicating parameters. Among those 13 species, four species belong to class Gastropod (Physella acuta and Mekongia crassa) and class - Bivalvia (Parreysia corrugata and Indonaia andersoniana); seven species belong to class Insecta (Anax sp. Dragonfly Nymph, Notonecta sp., Caenis sp., Rhyacophila sp., Philopotamus sp. and Chironomus larvae); and two species belong to class Clitellata (Tubifex tubifex and Lumbriculus variegatus). The strong negative correlation of Mekongia crassa with total phosphate indicated that the species can be marked as an essential aquatic riverine indicator species. The present study can conclude that these identified benthic species can be used as bioindicator organisms for pollution monitoring and riverine health.
\end{abstract}

Keywords: Pollution; Bioindicators; Macrobenthic fauna; Ecophysiological parameters; Ganga

\section{Introduction}

Bioindicators are referred to the accumulation of all the biological and physicochemical reactions in the ecological platform. Benthic organisms, settled in the sediment of an aquatic ecosystem are regarded as the key indicating species for environmental pollution. Aquatic environment vandalism is primarily noticed due to the consequences of hazards on the ecosystem [1-3]. Conventional methods of water quality analysis have been taking place through repeated sampling procedures, but this may not be a precise way to conclude the quality until one combines the abiotic factor with the biotic community. Bioindicating species are rudimentary organisms that can thrive in a planktic or benthic habitat of the aquatic ecosystem. The Ganga river system is one of the oldest and most primitive riverine flowing through extensive land is under constant monitoring to maintain the river health [4]. A constant observational study was carried out on the physicochemical as well as the biological parameters of the river mainly biological oxygen demand (BOD), chemical oxygen demand (COD), dissolved oxygen (DO), pH, water temperature, total coliforms, faecal coliforms etc. [4]. Hydrological and chemical alterations have a prolonged effect on the riverine biota that could be accessed through monitoring surveys [5]. The aquatic ecosystem is a valuable asset to humans as it constitutes more than $71 \%$ of the Earth and comprises $90 \%$ biodiversity of the biosphere [6].

Thus, benthic organisms are commonly considered as bioindicators to assess the pollution impacts of the aquatic environment. Numerous authors have depicted the beneficial usage of macrobenthic organisms for biological assessment in the natural environment [7]. Alterations in the chemical composition of water and sediment along with a change in primary productivity can have a greater effect on the sedentary community which leads to a decrease in diversity or escaping off the community species along with mass mortality and physiological interchanges [3]. Precise differences in the physiological activities like feeding, locomotion and life cycle depict the sensitivity of the benthic individuals to tolerate certain types of pollution [8]. Through the changes that occurred in the natural system, due to contamination, we can predict the degree of pollution by studying these organisms [9]. 
Materials and Methods

\section{Details of study area}

The study area comprises of the entire stretch of river Ganga starting from Harsil (upper Ganges) to Fraserganj (lower Ganges) (Figure 1). Twenty stations covering from Uttrakhand, Uttar Pradesh to West Bengal via Bihar are the targeted sampling sites along the river. The sampling stations were divided into the upper zone (Harshil to Farukhabad), middle zone (Kanpur to Farakka), lower zone (Jangipur to Tribeni) and estuarine zone (Godakhali to Fraserganj). The bottom substratum of the sites varies from one zone to the other. The upper zone mostly comprises of rocky river bed while the middle part is comprised of clay bottom and the lower part is mostly composed of silt, clay and sand.

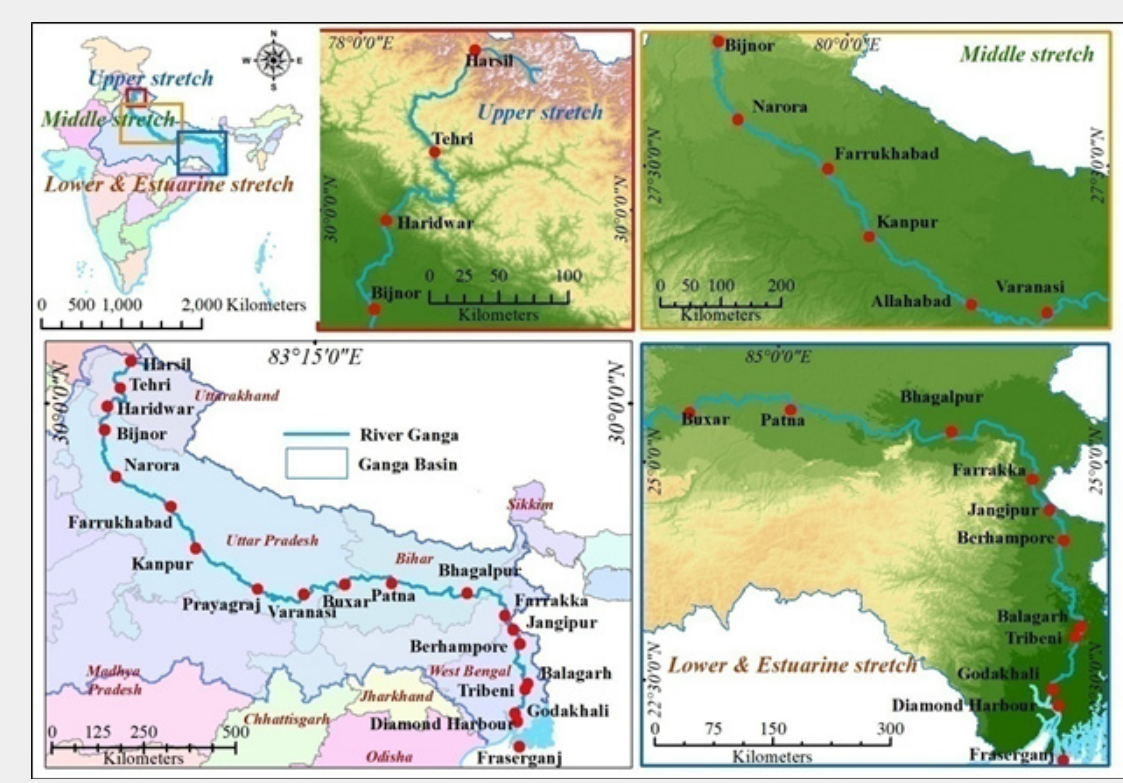

Figure 1: Study area along river Ganga though GIS mapping

\section{Collection, preservation and identification}

The macrobenthic species were collected with the help of Peterson Garb ( area $=2.31 \mathrm{~m}^{2}$ ) while the larger molluscan species were collected by handpicking. The collected muddy samples in the grab were mixed with water in a bucket and sieved. The samples were sieved through $500 \mu \mathrm{m}$ and $63 \mu \mathrm{m}$ for diversity sorting. The sieved samples were preserved in sterile sample bottle with 4\% formalin. Sorting of Annelid species was carried out with the help of stereomicroscope (ZEISS Semi 2000-C). Taxonomic identification of different macrobenthic organisms was performed with the help of literature [10-14].

\section{Water and sediment chemistry}

For water quality parameters, the samples was collected in sterilized sample bottles from each sampling station. Nutrient parameters like total phosphate (WTP) (mg/l or ppm) and total nitrogen (WTN) (mg/l or ppm); total chlorophyll through acetone extraction procedure (TC) (mg/l or ppm) and soil organic carbon (SOC) (\%) was analyzed by following the standard methodology and protocol mentioned in [15].

\section{Statistical analysis}

Karl Pearson's correlation between pollution indicating parameters and benthic samples were analyzed through multivariate software IBM-SPSS Statistics 22. The diversity variants was analyzed through PAST software version 4.2.

\section{Results}

\section{Hydrographic parameters}

The indicating parameters, which are considered during the experimental study at twenty different stations, are total phosphate, total nitrogen, total chlorophyll and soil organic carbon is depicted in Table 1. The maximum amount of total phosphate was observed at Bijnor (1.306ppm), while the minimum was recorded at Balagarh and Tribeni $(0.054 \mathrm{ppm})$ respectively. The maximum total nitrogen was recorded at Kanpur (4.750ppm), while the minimum was recorded at Fraserganj $(0.506 \mathrm{ppm})$. The total chlorophyll showed a diverse range with maximum recorded at Prayagraj (169.812ppm) and minimum at Harsil (0.823ppm). Likewise, the soil organic carbon recorded maximum at Varanasi (1.465ppm) and minimum at Harsil (0.193ppm).

\section{Macro-benthic faunal diversity of river Ganga}

A total of 69 species (Figure 2) has been recorded along the entire river stretch belonging to 3 Phyla and 4 different classes viz. Mollusca (Gastropoda and Bivalvia), Arthropoda (Insecta) and Annelida (Clitellata). There are 5 different groups of organisms found along the stretch of river Ganga, freshwater 
gastropods, brackishwater gastropods, freshwater bivalves, insects and clitellata. Amongst these, 18 species belong to freshwater gastropods which are largely available from Haridwar to Godakhali; 13 species belonging to brackishwater gastropods primarily from Diamond Harbour and Fraserganj; 13 freshwater bivalve species found from Haridwar to Diamond Harbour. 20 species belonging to the insects group were recorded from Harsil to Jangipur and 4 species from clitellata were recorded from Harsil to Fraserganj. The maximum diversity was observed in case of class gastropod viz. Filopaludina bengalensis, Melanoides tuberculata, Assiminae francesiae, Brotia costula, Tarebia lineata, Tarebia granifera, Gyraulus convexiusculus, Gabbia orcula and Lymnaea acuminata. Neripteron violaceum belonging to estuarine gastropod is one of the dominant species in Fraserganj. Class Bivalvia depicts the dominance of four species primarily; Parreysia favidens, P. corrugata, P. occata and Corbicula striatella. Chironomid larvae are the dominant species belonging to class Insecta. The distribution of macrofaunal diversity across river Ganga is shown in Table 2.

Table 1: Hydrological parameters of all the twenty stations depicting their maximum and minimum values expressed in mean \pm SD.

\begin{tabular}{|c|c|c|c|c|c|c|c|c|c|c|c|c|}
\hline Stations & Max & Min & WTP & Max & Min & WTN & Max & Min & WTC & Max & Min & SOC \\
\hline Harsil & 0.379 & 0.067 & $0.243 \pm 0.13$ & 1.45 & 0.31 & $0.848 \pm 0.46$ & 2.136 & 0 & $0.823 \pm 1.00$ & 0.38 & 0.018 & $0.193 \pm 0.14$ \\
\hline Tehri & 0.384 & 0.098 & $0.213 \pm 0.12$ & 4.461 & 0.912 & $2.537 \pm 1.60$ & 11.748 & 2.136 & $8.01 \pm 4.31$ & 2.231 & 0.474 & $1.368 \pm 0.71$ \\
\hline Haridwar & 1.374 & 0.594 & $1.029 \pm 0.38$ & 6.608 & 1.546 & $3.813 \pm 2.53$ & 30.972 & 2.136 & $11.214 \pm 13.46$ & 1.287 & 0.091 & $0.825 \pm 0.51$ \\
\hline Bijnor & 2.939 & 0.259 & $1.306 \pm 1.14$ & 6.398 & 0.115 & $3.351 \pm 3.04$ & 18.262 & 4.272 & $9.9057 \pm 6.47$ & 2.061 & 0.128 & $1.177 \pm 0.79$ \\
\hline Narora & 1.662 & 0.315 & $0.875 \pm 0.56$ & 3.134 & 1.023 & $2.471 \pm 0.99$ & 91.848 & 3.204 & $30.171 \pm 41.37$ & 1.155 & 0.155 & $0.833 \pm 0.45$ \\
\hline $\begin{array}{c}\text { Far- } \\
\text { rukhabad }\end{array}$ & 1.375 & 0.308 & $0.779 \pm 0.48$ & 2.388 & 0.065 & $1.05 \pm 0.97$ & 37.38 & 1.068 & $14.952 \pm 15.84$ & 0.851 & 0.048 & $0.374 \pm 0.34$ \\
\hline Kanpur & 1.616 & 0.485 & $0.979 \pm 0.56$ & 9.006 & 1.569 & $4.750 \pm 3.10$ & 264.864 & 3.204 & $\begin{array}{c}135.636 \pm \\
110.76\end{array}$ & 1.202 & 0.107 & $0.810 \pm 0.48$ \\
\hline Prayagraj & 1.976 & 0.62 & $1.224 \pm 0.60$ & 4.518 & 0.368 & $2.807 \pm 2.05$ & 335.352 & 6.408 & $\begin{array}{c}169.812 \pm \\
157.79\end{array}$ & 2.635 & 0.773 & $1.386 \pm 0.84$ \\
\hline Varanasi & 1.351 & 0.694 & $1.022 \pm 0.29$ & 3.728 & 0.907 & $2.282 \pm 1.29$ & 389.82 & 9.612 & $\begin{array}{c}164.472 \pm \\
176.03\end{array}$ & 2.74 & 0.73 & $1.465 \pm 0.89$ \\
\hline Buxar & 0.24 & 0.062 & $0.139 \pm 0.08$ & 0.983 & 0.634 & $0.794 \pm 0.16$ & 8.334 & 3.26 & $6.2185 \pm 2.14$ & 0.523 & 0.367 & $0.461 \pm 0.07$ \\
\hline Patna & 0.126 & 0.054 & $0.106 \pm 0.03$ & 1.1 & 0.5 & $0.809 \pm 0.26$ & 8.74 & 3.4 & $5.67025 \pm 2.26$ & 0.365 & 0.24 & $0.301 \pm 0.06$ \\
\hline Bhagalpur & 0.466 & 0.113 & $0.208 \pm 0.17$ & 1.7 & 0.9 & $1.293 \pm 0.34$ & 11.627 & 2.24 & $6.34925 \pm 4.13$ & 0.585 & 0.285 & $0.503 \pm 0.14$ \\
\hline Farraka & 0.262 & 0.035 & $0.122 \pm 0.10$ & 2.2 & 0.5 & $1.011 \pm 0.79$ & 6.53 & 1.73 & $4.1755 \pm 1.97$ & 0.78 & 0.495 & $0.608 \pm 0.13$ \\
\hline Jangipur & 0.167 & 0.02 & $0.070 \pm 0.06$ & 1.4 & 0.3 & $0.950 \pm 0.49$ & 5.36 & 2.74 & $4.3255 \pm 1.12$ & 0.553 & 0.27 & $0.376 \pm 0.12$ \\
\hline $\begin{array}{l}\text { Behram- } \\
\text { pur/ } \\
\text { Rejinagar }\end{array}$ & 0.146 & 0.043 & $0.099 \pm 0.04$ & 0.9 & 0.5 & $0.666 \pm 0.20$ & 24.26 & 3.49 & $10.285 \pm 9.68$ & 0.485 & 0.155 & $0.362 \pm 0.15$ \\
\hline Balagarh & 0.081 & 0.039 & $0.054 \pm 0.01$ & 0.8 & 0.5 & $0.608 \pm 0.13$ & 21.01 & 11.17 & $14.852 \pm 4.65$ & 0.755 & 0.195 & $0.522 \pm 0.25$ \\
\hline Tribeni & 0.067 & 0.044 & $0.054 \pm 0.01$ & 1 & 0.3 & $0.567 \pm 0.31$ & 52.948 & 7.49 & $20.5445 \pm 21.66$ & 0.75 & 0.53 & $0.640 \pm 0.11$ \\
\hline Godakhali & 0.233 & 0.035 & $0.110 \pm 0.09$ & 2.5 & 0.5 & $1.24 \pm 0.87$ & 32.75 & 5.25 & $16.655 \pm 12.20$ & 0.693 & 0.375 & $0.475 \pm 0.14$ \\
\hline D. Harbour & 0.144 & 0.042 & $0.091 \pm 0.05$ & 1.5 & 0.4 & $0.746 \pm 0.49$ & 19.58 & 2.38 & $12.849 \pm 7.72$ & 0.892 & 0.355 & $0.695 \pm 0.23$ \\
\hline Fraserganj & 0.137 & 0.026 & $0.071 \pm 0.04$ & 0.8 & 0.33 & $0.506 \pm 0.22$ & 19.3 & 3.24 & $12.9785 \pm 7.15$ & 0.937 & 0.39 & $0.768 \pm 0.25$ \\
\hline
\end{tabular}

Correlation between benthic Gangetic species and physicochemical parameters

Karl Pearson's correlation coefficient computed between the pollution indicating ecological parameters and the available species across the riverine stretch, showed a highly significant variation and correlation $(\mathrm{p}<0.01$ and $\mathrm{p}<0.05)$. The correlation within the assigned parameters showed a high significant variation between each other. WTN positively correlated to WTP $(r=0.898, p<0.01)$, while WTC showed significant positive correlation with both WTP and WTN $(r=0.662, p<0.01 ; r=0.687$, $\mathrm{p}<0.01)$. SOC showed a significant positive coefficient ' $r$ ' values for WTP, WTN and WTC $(\mathrm{r}=0.814, \mathrm{p}<0.01 ; \mathrm{r}=0.865, \mathrm{p}<0.01 ; \mathrm{r}$ $=0.688, \mathrm{p}<0.01$ ). Correction coefficients between 13 species and environmental parameters were depicted in table 3 , showing both positive and negative relation within the aforesaid parameters.

\section{Canonical correspondence analysis}

Canonical correspondence analysis in Figure 3 depicts the inter relationship between the ecological parameters and the macro-benthic invertebrates. Through the CCA biplot, an 
eigenvalue of 0.147 and 0.111 was calculated on axis 1 and axis 2 with a respective percentage value of 0.167 and 0.137 . The plot reveals that the four pollution indicating parameters showed different correlations with the macro-benthic community. Chironomid larvae along with Notonecta sp. and Philopotamus sp. were influenced by WTC, while WTN and WTP highly influence and show a positive gradient towards Parreysia corrugata, Dragonfly nymph, Anax sp., Indonaia andersoniana. Soil organic carbon influences Physella acuta, Lumbriculus variegates, Rhyacophila sp., Caenis sp.

Table 2: Distribution pattern of the available macrozoobenthic species at twenty different stations.

\begin{tabular}{|c|c|c|c|c|c|c|c|c|c|c|c|c|c|c|c|c|c|c|c|c|c|}
\hline$\frac{\hat{x}}{\tilde{v}}$ & 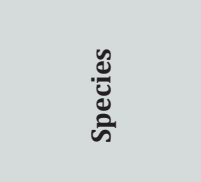 & 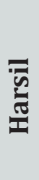 & 를 & 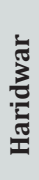 & 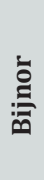 & 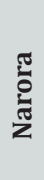 & 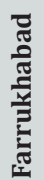 & 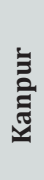 & 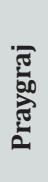 & 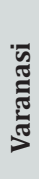 & 䒕 & $\overbrace{}^{\pi}$ & 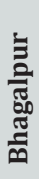 & $\frac{\pi}{\frac{\pi}{x}}$ & 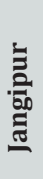 & 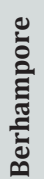 & 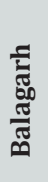 & 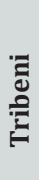 & 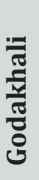 & 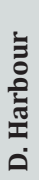 & 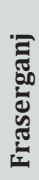 \\
\hline \multirow{18}{*}{$\begin{array}{c}\text { Gastropoda } \\
\text { (Freshwater) }\end{array}$} & Physella acuta & - & - & + & + & + & + & + & + & + & - & + & - & - & - & - & - & - & - & - & - \\
\hline & $\begin{array}{c}\text { Filopaludina } \\
\text { bengalensis }\end{array}$ & - & - & - & + & + & + & + & + & + & + & + & + & + & + & + & + & + & + & + & + \\
\hline & $\begin{array}{l}\text { Idiopoma dissi- } \\
\text { milis }\end{array}$ & - & - & - & - & - & - & - & - & - & - & + & + & - & - & - & - & - & - & - & - \\
\hline & $\begin{array}{l}\text { Mieniplotia } \\
\text { scabra }\end{array}$ & - & - & - & - & + & - & - & + & + & + & - & + & - & - & - & - & - & - & - & - \\
\hline & $\begin{array}{l}\text { Mekongia } \\
\text { crassa }\end{array}$ & - & - & - & - & - & - & - & - & - & + & + & - & + & + & + & + & - & - & - & - \\
\hline & $\begin{array}{l}\text { Melanoides } \\
\text { tuberculata }\end{array}$ & - & - & - & + & + & + & + & + & + & + & + & + & + & + & + & + & + & - & + & + \\
\hline & $\begin{array}{l}\text { Assiminae fran- } \\
\text { cesiae }\end{array}$ & - & - & - & - & - & - & - & - & - & - & - & - & - & - & - & - & + & + & + & - \\
\hline & Brotia costula & - & - & - & + & + & + & + & + & + & + & + & + & + & + & + & + & + & - & - & + \\
\hline & Tarebia lineata & - & - & - & + & + & + & + & + & + & - & + & - & + & - & + & + & + & + & - & + \\
\hline & $\begin{array}{l}\text { Tarebia gran- } \\
\quad \text { ifera }\end{array}$ & - & - & - & - & - & - & - & - & - & + & + & + & + & + & + & + & + & + & + & + \\
\hline & Gyraulus parvus & - & - & - & - & - & - & - & - & - & + & - & - & + & - & - & - & - & + & - & - \\
\hline & $\begin{array}{l}\text { Gyraulus con- } \\
\text { vexiusculus }\end{array}$ & - & - & + & + & + & + & + & + & + & - & - & - & + & - & - & - & - & - & - & - \\
\hline & Gyraulus rotula & - & - & - & + & - & - & - & - & - & - & - & - & - & - & - & - & - & - & - & - \\
\hline & $\begin{array}{l}\text { Lymnaea acumi- } \\
\text { nata }\end{array}$ & - & - & + & + & + & + & + & + & + & - & - & + & + & + & - & - & - & - & + & - \\
\hline & Racesina luteola & - & - & - & - & - & - & - & - & - & - & + & + & - & - & - & - & - & - & - & - \\
\hline & Gabbia orcula & - & - & - & - & - & - & - & - & - & - & + & + & + & - & - & - & - & + & - & - \\
\hline & $\begin{array}{l}\text { Indoplanorbis } \\
\text { exustus }\end{array}$ & - & - & - & - & - & - & - & - & - & - & - & + & + & - & - & - & - & - & - & - \\
\hline & Pila globossa & - & - & - & - & - & - & - & - & - & - & - & - & + & + & - & + & - & - & - & - \\
\hline
\end{tabular}


International Journal of Environmental Sciences \& Natural Resources

\begin{tabular}{|c|c|c|c|c|c|c|c|c|c|c|c|c|c|c|c|c|c|c|c|c|c|}
\hline \multirow{13}{*}{$\begin{array}{c}\text { Gastropoda } \\
\text { (Brackish- } \\
\text { water) }\end{array}$} & $\begin{array}{c}\text { Stenomelania } \\
\text { plicaria }\end{array}$ & - & - & - & - & + & - & - & - & + & - & - & - & - & - & - & - & - & - & - & - \\
\hline & Nerita balteata & - & - & - & - & - & - & - & - & - & - & - & - & - & - & - & - & - & - & - & + \\
\hline & Nerita fulgurans & - & - & - & - & - & - & - & - & - & - & - & - & - & - & - & - & - & - & - & + \\
\hline & $\begin{array}{l}\text { Neripteron } \\
\text { violaceum }\end{array}$ & - & - & - & - & - & - & - & - & - & - & - & - & - & - & - & - & - & - & + & - \\
\hline & Vittina smithii & - & - & - & - & - & - & - & - & - & - & - & - & - & - & - & - & - & - & + & - \\
\hline & $\begin{array}{l}\text { Volegalea coch- } \\
\text { lidium }\end{array}$ & - & - & - & - & - & - & - & - & - & - & - & - & - & - & - & - & - & - & - & + \\
\hline & $\begin{array}{l}\text { Cerithidea } \\
\text { obtusa }\end{array}$ & - & - & - & - & - & - & - & - & - & - & - & - & - & - & - & - & - & - & - & + \\
\hline & $\begin{array}{l}\text { Pirenella cin- } \\
\text { gulata }\end{array}$ & - & - & - & - & - & - & - & - & - & - & - & - & - & - & - & - & - & - & - & + \\
\hline & $\begin{array}{l}\text { Telescopium } \\
\text { telescopium }\end{array}$ & - & - & - & - & - & - & - & - & - & - & - & - & - & - & - & - & - & - & - & + \\
\hline & $\begin{array}{l}\text { Nassarius } \\
\text { stolatus }\end{array}$ & - & - & - & - & - & - & - & - & - & - & - & - & - & - & - & - & - & - & - & + \\
\hline & $\begin{array}{l}\text { Nassarius fove- } \\
\text { olatus }\end{array}$ & - & - & - & - & - & - & - & - & - & - & - & - & - & - & - & - & - & - & - & + \\
\hline & Ancilla ampla & - & - & - & - & - & - & - & - & - & - & - & - & - & - & - & - & - & - & - & + \\
\hline & Murex tribulus & - & - & - & - & - & - & - & - & - & - & - & - & - & - & - & - & - & - & - & + \\
\hline \multirow{13}{*}{$\begin{array}{c}\text { Bivalvia } \\
\text { (Freshwater) }\end{array}$} & $\begin{array}{l}\text { Novaculina } \\
\text { gangetica }\end{array}$ & - & - & - & + & + & + & + & + & + & + & + & + & - & - & - & - & + & - & - & - \\
\hline & $\begin{array}{l}\text { Lamellidens } \\
\text { corrianus }\end{array}$ & - & - & - & + & + & + & + & + & + & + & + & + & + & - & - & - & - & - & - & - \\
\hline & $\begin{array}{c}\text { Lamellidens } \\
\text { marginalis }\end{array}$ & - & - & - & - & - & - & - & - & - & - & + & - & - & - & - & - & - & - & - & - \\
\hline & $\begin{array}{l}\text { Parreysia } \\
\text { favidens }\end{array}$ & - & - & - & + & + & + & + & + & + & + & + & + & + & + & + & + & - & - & - & - \\
\hline & $\begin{array}{l}\text { Parreysia cor- } \\
\quad \text { rugata }\end{array}$ & - & - & + & + & + & + & + & + & - & - & - & - & - & - & - & - & - & - & - & - \\
\hline & $\begin{array}{c}\text { Parreysia shur- } \\
\text { tleffiana }\end{array}$ & - & - & - & - & - & - & - & - & - & - & + & + & - & - & - & - & - & - & - & - \\
\hline & $\begin{array}{l}\text { Parreysia } \\
\text { occata }\end{array}$ & - & - & - & - & - & - & - & - & - & + & + & - & + & - & - & + & - & - & - & - \\
\hline & Parreysia lima & - & - & - & - & - & - & - & - & - & + & - & - & - & - & - & - & - & - & - & - \\
\hline & $\begin{array}{l}\text { Indonaia ander- } \\
\text { soniana }\end{array}$ & - & - & - & + & + & - & - & + & - & - & - & - & - & - & - & - & - & - & - & - \\
\hline & $\begin{array}{l}\text { Parreysia an- } \\
\text { nandalei }\end{array}$ & - & - & - & - & + & - & - & - & - & - & - & - & - & - & - & - & - & - & - & - \\
\hline & $\begin{array}{c}\text { Parreysia } \\
\text { caerulea }\end{array}$ & - & - & - & - & - & - & - & - & - & - & - & - & + & - & - & - & - & - & - & - \\
\hline & $\begin{array}{l}\text { Corbicula stri- } \\
\text { atella }\end{array}$ & - & - & - & + & + & + & + & + & + & + & + & + & + & - & - & - & - & - & + & - \\
\hline & $\begin{array}{c}\text { Corbicula } \\
\text { bensoni }\end{array}$ & - & - & - & - & - & - & - & - & - & - & + & - & - & - & - & + & - & - & - & - \\
\hline
\end{tabular}


International Journal of Environmental Sciences \& Natural Resources

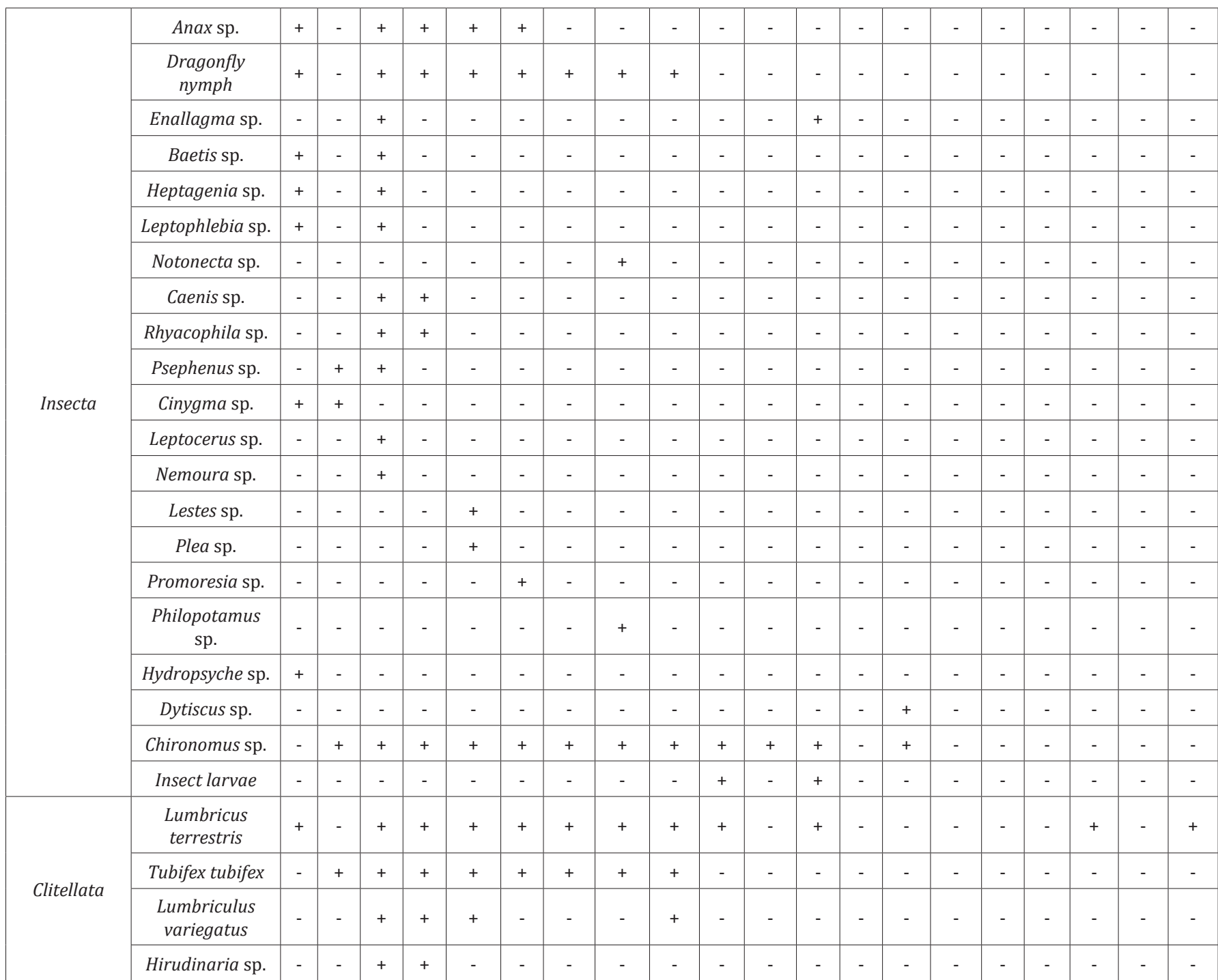

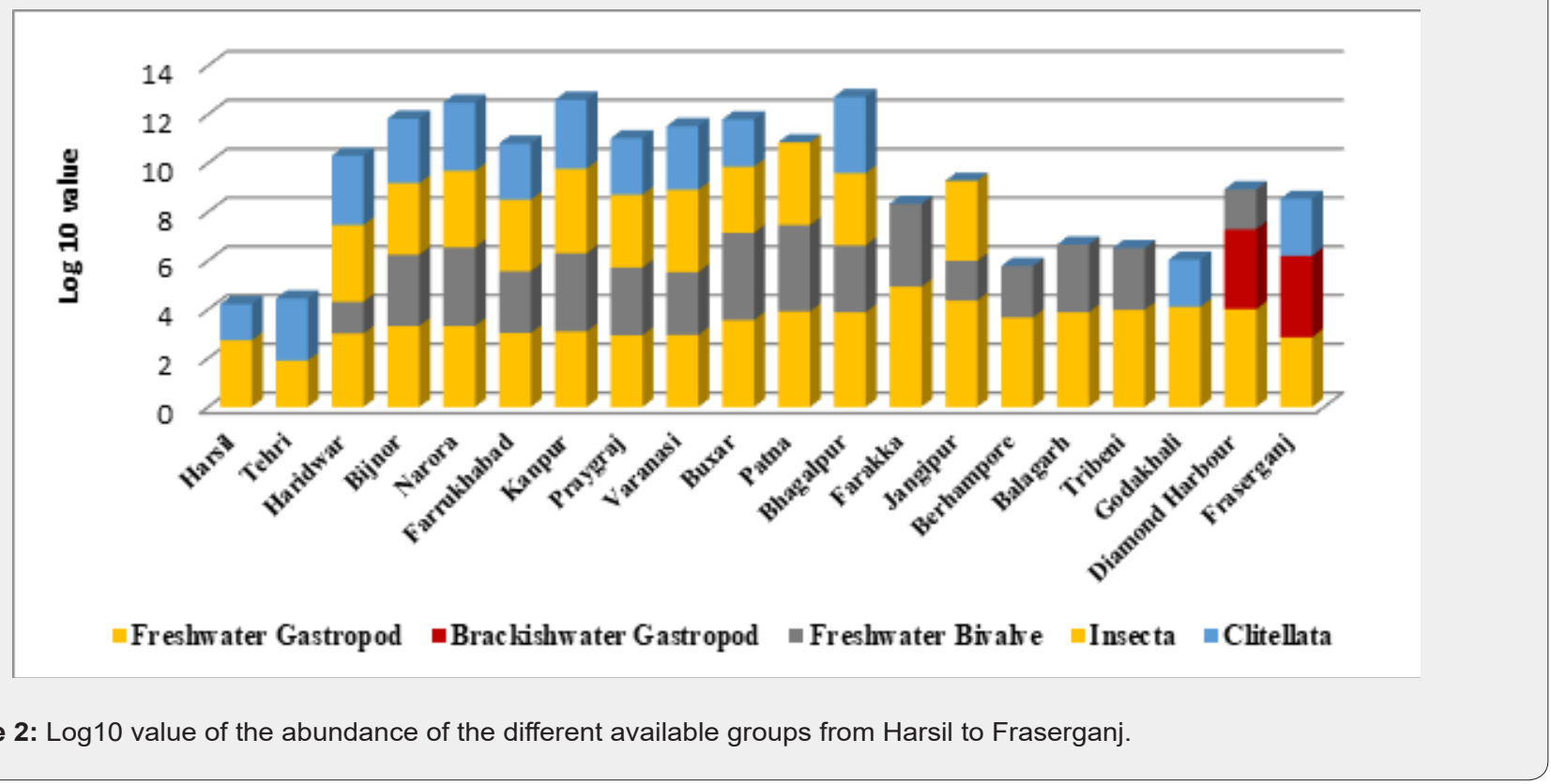




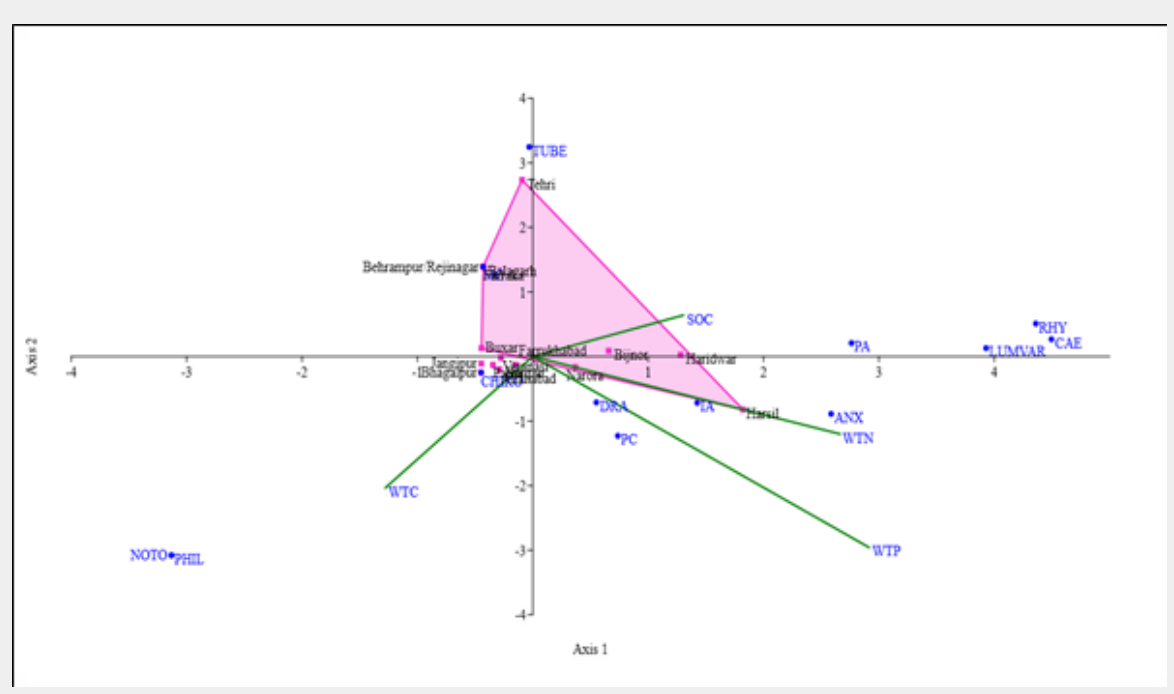

Figure 3: Canonical correspondence analysis between ecological parameters and macrobenthic organisms.

Table 3: Correlation between physicochemical parameters and available species collected from the river Ganga.

\begin{tabular}{|c|c|c|c|c|c|c|c|c|c|c|c|c|c|}
\hline & PhyA & MekoC & ParreC & IndoA & Anx & Dra & Noto & Cae & Rhy & Phil & Chiro & Tube & LumV \\
\hline WTP & - & $-.446^{*}$ & $.530^{*}$ & $.473^{*}$ & $.504^{*}$ & $.675^{* *}$ & - & $.502^{*}$ & $.536^{*}$ & - & - & $.578^{* *}$ & $.510^{*}$ \\
\hline WTN & $.461^{*}$ & - & $.589 * *$ & - & - & $.676^{* *}$ & - & - & $.481^{*}$ & - & $.480^{*}$ & $.766^{* *}$ & - \\
\hline WTC & - & - & - & - & - & $.489 *$ & $.594^{* *}$ & - & - & $.594^{* *}$ & $.563^{* *}$ & $.466^{*}$ & - \\
\hline SOC & - & - & - & - & - & $.518^{*}$ & - & - & - & 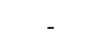 & - & $.768^{* *}$ & - \\
\hline
\end{tabular}

** Correlation is significant at the 0.01 level (2-tailed).

${ }^{*}$ Correlation is significant at the 0.05 level (2-tailed).

\section{Discussion}

River Ganga covering a vast stretch of land from north to southeast across India, comprises a diversified species distribution along its course. The optimum physico chemical parameters of the river facilitate in the sustainability of the benthic organisms.

Total nitrogen, in the present study, showed a positive correlation with six macrobenthic species (Physella acuta, Parreysia corrugata, Dragonfly nymph, Rhyacophila sp., Chironomid larvae and Tubifex tubifex) with a significance level ( $r=0.461,0.480$ and $0.481, \mathrm{p}<0.05)$. It has been reported that in the anthropogenic polluted areas, isotopes of nitrogen $\left(\delta^{15} \mathrm{~N}\right)$ acts as a biological indicator for aquatic ecosystem from primary producers to invertebrates and fish [16-20]. A significant positive correlation was observed between nitrate content of water and gastropod species density and richness in lentic waterbodies of East Kolkata wetlands and Damodar basin [21,22]. The present work shows phosphate is positively correlated to macrobenthic faunal diversity like Parreysia corrugata, Indonaia andersoniana, Anax sp., Dragonfly nymph, Rhyacophila sp., Caenis sp, Tubifex tubifex and Lumbriculus variegatus. Phosphate is a major catalyst for eutrophication and act as a limiting factor for the growth of the benthic organism in any aquatic body [22], yet it promotes substantial growth for benthic organisms. The total phosphate of water showed a significant negative correlation with Mekongia crassa $(r=-0.446, p<0.05)$ denoting clearly that this species grows well in a moderately polluted area. To validate the study, a reference of [23] is presented that denotes Mekongia crassa, a gastropod of viviparidae family, is recorded in Class B of the water quality indicators in river Ganga. The contribution of soil organic carbon and total chlorophyll was also found to be significant during the whole study. According to [24], soil organic carbon influences the distribution pattern and abundance of the benthic species especially annelids and molluscan groups. The correlation between soil organic carbon and dragonfly nymph showed a significant ' $r$ ' value of $0.518, \mathrm{p}<0.05$.

In CCA biplot analysis, the macrobenthic assemblage showed more gradient towards total nitrogen (WTN), total phosphate (WTP) and soil organic carbon (SOC) establishing that these parameters help in the growth of the aquatic organisms at Harsil, Bijnor, Narora, Haridwar and Farrukhabad. The above selected 13 species act as bioindicator species to analyse the quality of the riverine water.

A freshwater invasive species of gastropod, Physella acuta, was reported to be a potential indicator for bioaccumulation of heavy metals primarily $\mathrm{Cu}$ and $\mathrm{Zn}$ [25]. Since this species is tolerant to moderate - heavy pollution, it can survive in the natural 
environment as well as in the industrial polluted areas [26]. The abundance of this species in the present research was recorded maximum in the upper stretch of the river Ganga from Haridwar to Varanasi and showed a positive correlation with total nitrogen. P. acuta is acclimatized from clean water to heavily polluted areas in the river Ganga. Thus, it has been categorised from Class A to D in the water quality indicators in river Ganga [23]. Parreysia corrugata, is regarded as one of the highly priced edible mussels cultured in India. This bivalve species shows a positive correlation with WTN and WTP denoting that these organisms are more gradient towards the nutrient rich quality of water. This organism prefers a slightly moderate polluted zone in river Ganga denoting a Class C section of the water quality indicators [23]. Among the seven insect species recorded as bioindicators across the Ganga river, dragonfly showed the maximum positive significance with all the aforesaid parameters. Dragonfly nymph is a common aquatic insect that spends part of its life cycle under water. Studies are showing the diversity of dragonfly nymph was dominated in a clean and healthy riverine ecosystem. Early changes in the environmental conditions are highly depicted by dragonfly nymphs as they are vulnerable to slight alterations in the ecosystem. Some of the species of dragonfly like Boyeria irene and Cordulegaster boltonii are highly intolerant to pollution [27]. The non existence of certain dragonfly species like Calopteryx sp. and Oxygastra sp. across the Guadiamar river suggested the ecological degradation of the aquatic ecosystem [28]. Anax sp. is another species of dragonfly, frequently reported in the upper stretch from Harshil to Farrukhabad where the water is partially polluted. Other insects like Caenis sp., Rhyacophila sp., Notonecta sp. and Philopotamus sp. also show a positive gradient towards WTC, WTN and WTP.

In the current study, chironomid larvae also showed a remarkable influence with four pollution indicating parameters. Chironomid larvae showed a positive correlation with WTN ( $\mathrm{r}$ $=0.480, \mathrm{p}<0.05$ ). In inland water bodies, species belonging to Chironomidae family are considered as indicators of degraded and poor quality of water [29]. Chironomus riparius is a common freshwater chironomid species, which shows evolutionary deformities when exposed to metal contaminations and responds rapidly to external malfunctions [29,30]. Therefore, though they can flourish in a contaminated ecosystem, their anatomical deformities established that a few of the traits might extinct during the process of their habitat adaptation. Another important species belonging to phylum Annelid and class Clitellata is Tubifex tubifex which has a prime function in maintaining the soil influx and nutrient development. This might be the reason that this particular organism has a positive correlation with all the above mentioned parameters.

\section{Conclusion}

The benthic macroinvertebrates are the important species in the sediment of any ecosystem. They help in the sediment influx and soil fertility by churning the sediment particles with the help of their peristaltic movements. The increase in any of the nutrient parameters causes an imbalance in the ecosystem leading to the growth of a particular species and demolishing the population of other species. There are precise pros and cons of a nutrient rich environment for survivability, one must accept the benefits rather than causing hazard to the ecosystem. The interrelationship between the physicochemical parameters and the macrozoobenthic fauna can determine the assemblage and distribution patterns which in turn can further play an important role for the ecologist to analyse and assess the aquatic ecosystem for future.

\section{Acknowledgement}

We humbly thank the National Mission for Clean Ganga (NMCG), Ministry of Jal Sakti, Govt. of India, for providing us a platform to perform the research experiments along the Ganga river system and financially supporting us to perform the task. (Project No. T-17/2014-15/526/NMCG-Fish and Fisheries).

\section{Author Contribution}

Conceptualisation, supervison and final editing, Dr. Basanta Kumar Das; Validation and analysis, Canciyal Johnson and Himanshu Sekhar Swain; Sample collection, analysis and manuscript preparation, Shreya Roy, Supriti Bayen, Archisman Ray Trupti Rani Mohanty and Manisha Bhor.

\section{Declaration}

Consent to participate: All the authors consented towards the preparation of this manuscript.

Consent for publication: All the authors consented towards the publication of this manuscript.

Conflict of Interest: The authors declared that there are no conflicts of interest.

Ethical Approval: Not Applicable.

Availability of data and materials: Not Applicable.

\section{References}

1. Nybakken JW (1988) Marine Biology an Ecological Approach. Harper and Row Publisher, New York, USA 5: 443-446.

2. Steigerwalt NM (2005) Environmental factors affecting aquatic invertebrate community structure on snags in the Ichetucknee River, Florida, (Doctoral dissertation, University of Florida).

3. Uwadiae RE (2016) Benthic macroinvertebrate community and chlorophyll a (chl-a) concentration in sediment of three polluted sites in the Lagos Lagoon, Nigeria. Journal of Applied Sciences and Environmental Management 20(4): 1137-1145.

4. Agrawal S, Sharma J, Goel A (2019) Bioassessment of river ganga in Uttarakhand stretch (India) using benthic macro-invertebrates as bioindicator. Applied Biological Research 21(3): 235-244.

5. Akolkar P, Sharma J, Goel A, Ahmad I, Ahmad F (2017a) Method of assessment of biological health of River Ganga. Biological Health of River Ganga. Central Pollution Control Board, Ministry of Environment, Forest \& Climate Change, New Delhi, India, pp. 4-5. 
6. Scot F (2005) Biological Science. Pearson Education, Inc.

7. Hellawell JM (2012) Biological indicators of freshwater pollution and environmental management. Springer Science \& Business Media 1: $1-546$.

8. Rosenberg DM, Resh VH (1993) Freshwater biomonitoring and benthic macroinvertebrates. Chapman and Hall, London.

9. Khatri N, Tyagi S (2015) Influences of natural and anthropogenic factors on surface and ground water quality in rural and urban areas. Frontiers in Life Science 8(1): 23-39.

10. Needham JG (1957) $A$ guide to the study Freshwater Biology with special reference of Aquatic Insects and other Invertebrates Animals and Phytoplankton. Comstock 4: 1-88.

11. Edmondson WT (1959) Freshwater Biology. pp. 1-1248.

12. Brinkhurst RO (1971) A guide for the identification of British Aquatic Oligochaeta. Ambleside \& Westmorland, pp. 1-58.

13. Warwick RM, Platt HM, Somerfield PJ (1998) Free-living marine nematodes: part III. Monhysterida. Synopses of the British Fauna no. 53. Field Studies Council.

14. Dey RA (2007) Handbook on Indian Freshwater Molluscs. Zoological Survey of India 1: 1-399.

15. APHA (2017) In: Baird RB, Eaton AD, Rice EW, Clesceri LS (Eds.) Standard Methods for the Examination of Water and Wastewater. American Public Health Association (APHA), American Water Works Association (AWWA) and Water Environment Federation (WEF) 23 1-1546.

16. Lake JL, McKinney RA, Osterman FA, Pruell RJ, Kiddon J, et al. (2001) Stable nitrogen isotopes as indicators of anthropogenic activities in small freshwater systems. Canadian Journal of Fisheries and Aquatic Sciences 58(5): 870-878.

17. Cole ML, Valiela I, Kroeger KD, Tomasky GL, Cebrian J, et al. (2004) Assessment of a $\delta^{15} \mathrm{~N}$ isotopic method to indicate anthropogenic eutrophication in aquatic ecosystems. Journal of Environmental Quality 33(1): 124-132.

18. Schlacher TA, Liddell B, Gaston TF, Schlacher-Hoenlinger M (2005) Fish track wastewater pollution to estuaries. Oecologia 144(4): 570584 .

19. Xu J, Xie P, Zhang M, Zhou Q, Zhang L, et al. (2007) Icefish (Salangidae) as an indicator of anthropogenic pollution in freshwater systems using nitrogen isotope analysis. Bulletin of Environmental Contamination and Toxicology 79(3): 323-326.
20.Xu J, Zhang M (2012) Primary consumers as bioindicator of nitrogen pollution in lake planktonic and benthic food webs. Ecological Indicators 14(1): 189-196.

21. Roy M, Nandi N, Banerjee S (2014) Macro-zoobenthic community and Assessment of Aquatic Ecosystem Health of three waterbodies of East Kolkata Wetlands, India. Proceedings of Zoological Society 67(2): 8693.

22. Ghosh P, Panigrahi AK (2018) A comprehensive study on correlation of gastropod diversity with some hydroenvironmental parameters of selected waterbodies of lower Damodar basin, West Bengal, India. Journal of Applied and Natural Science 10(4): 1259-1265.

23. Central Pollution Control Board (CPCB) Report (2017) Biological Indicators of Water Quality In River Ganga. Central Pollution Control Board, Ministry of Environment, Forest and Climate Change, Govt. of India, pp. 1-32.

24. Sharmin S, Rahman SH, Naser MN, Hoque S (2018) Macro benthic fauna in relation to limnological variables in a migratory bird visiting lake at Jahangirnagar University, Bangladesh. Journal of Biodiversity Conservation and Bioresource Management 4(2): 99-106.

25. Spyra A, Cieplok A, Strzelec M, Babczyńska A (2019) Freshwater alien species Physella acuta (Draparnaud, 1805) A possible model for bioaccumulation of heavy metals. Ecotoxicology and Environmental Safety 185: 109703 .

26. Jozwiak MA, Jozwiak M, Kozlowski R, Rabajczyk A (2010) The role of indicator malacofauna in pollution assessment of inland water exposed to anthropopressure: the case of the Kielce Lake. Ecological Chemistry and Engineering S 17(4): 485.

27. Martín R, Maynou X (2016) Dragonflies (Insecta: Odonata) as indicators of habitat quality in Mediterranean streams and rivers in the province of Barcelona (Catalonia, Iberian Peninsula). International Journal of Odonatology 19(3): 107-124.

28. Ferreras Romero M, Márquez Rodríguez J, Ruiz García A (2009) Implications of anthropogenic disturbance factors on the Odonata assemblage in a Mediterranean fluvial system. International Journal of Odonatology 12(2): 413-428.

29. Serra SR, Graça MA, Dolédec S, Feio MJ (2017) Chironomidae traits and life history strategies as indicators of anthropogenic disturbance. Environmental Monitoring and Assessment 189(7): 326.

30. Postma JF, Van Kleunen A, Admiraal W (1995) Alterations in lifehistory traits of Chironomus riparius (Diptera) obtained from metal contaminated rivers. Archives of Environmental Contamination and Toxicology 29(4): 469-475.

This work is licensed under Creative Commons Attribution 4.0 License DOI: 10.19080/IJESNR.2022.29.556273

\section{Your next submission with Juniper Publishers} will reach you the below assets

- Quality Editorial service

- Swift Peer Review

- Reprints availability

- E-prints Service

- Manuscript Podcast for convenient understanding

- Global attainment for your research

- Manuscript accessibility in different formats

( Pdf, E-pub, Full Text, Audio)

- Unceasing customer service

Track the below URL for one-step submission https://juniperpublishers.com/online-submission.php 